\title{
Pre-treatment with simvastatin prevents the induction of diet-induced atherosclerosis in a rabbit model
}

\author{
NIKOLAOS OIKONOMIDIS ${ }^{1}$, NIKOLAOS KAVANTZAS ${ }^{2}$, LASKARINA-MARIA KOROU ${ }^{1}$, \\ PANAGIOTIS KONSTANTOPOULOS ${ }^{1}$, VASILIOS PERGIALIOTIS ${ }^{1}$, EVANGELOS MISIAKOS ${ }^{3}$, \\ IOANNIS RIZOS ${ }^{4}$, CHRISTOS VERIKOKOS ${ }^{5}$ and DESPINA N. PERREA ${ }^{1}$
}

${ }^{1}$ Laboratory of Experimental Surgery and Surgical Research 'N.S. Christeas', National and Kapodistrian University of Athens, Medical School; ${ }^{2}$ 1st Department of Pathology, National and Kapodistrian University of Athens, Medical School,

Laiko University Hospital, Athens $11527 ;{ }^{3}$ 3rd Department of Surgery; ${ }^{4}$ 2nd Department of Cardiology,

National and Kapodistrian University of Athens, Medical School, Attikon University Hospital,

Athens 12462; ${ }^{5}$ 2nd Department of Propedeutic Surgery, National and Kapodistrian University

of Athens, Medical School, Laiko University Hospital, Athens 11527, Greece

Received August 16, 2016; Accepted September 19, 2016

DOI: 10.3892/br.2016.780

\begin{abstract}
The aim of the present study was to investigate the potential antiatherosclerotic activities of simvastatin in rabbits. Twenty-two, male, New Zealand rabbits were divided into the following groups: Control group (group C); cholesterol group (group A), in which the rabbits were fed a commercial rabbit chow supplemented with $0.5 \% \mathrm{w} / \mathrm{w}$ cholesterol for 8 weeks and then fed with normal chow for an additional 8 weeks; and a treatment group (group B), in which the rabbits initially received standard commercial rabbit chow along with being administered simvastatin for 8 weeks, following which they consumed a high-cholesterol diet for a further 8 weeks. The rabbits pre-treated with simvastatin presented significantly lower serum cholesterol and low-density lipoprotein cholesterol levels when compared with the non simvastatin-treated cholesterol-fed animals. Furthermore, none of the rabbits in the simvastatin group presented with atherosclerotic lesions in the aorta. Thus, simvastatin was demonstrated to exhibit preventive properties against the formation of atherosclerosis in the atherosclerosis model in the current study, predominantly via its hypolipidemic activity.
\end{abstract}

Correspondence to: Dr Nikolaos Oikonomidis, Laboratory of Experimental Surgery and Surgical Research 'N.S. Christeas', National and Kapodistrian University of Athens, Medical School, 15B Agiou Thoma, Athens 11527, Greece

E-mail:md.oikonomidis@gmail.com

Key words: atherosclerosis, simvastatin, cholesterol diet, rabbits, cholesterol

\section{Introduction}

Atherosclerosis is an inflammatory procedure characterized by the appearance of fatty deposits known as atheromatous plaques in the inner layers of arteries. The synergistic action of increased serum lipid levels, lipid peroxidation as well as disturbances in nitric oxide (NO) homeostasis and inflammation facilitate the initiation of atherosclerosis (1).

$\mathrm{CD} 163$ is a $130-\mathrm{kDa}$ member of the scavenger receptor cysteine rich (SRCR) family expressed on the surface of monocytes/macrophages. The soluble form of CD163 normally circulates in plasma, and is produced by the proteolytic cleavage of CD163 at the cell surface. CD163 is involved in the uptake of hemoglobin-haptoglobin and mediates the clearance of hemoglobin (2). Patients with severe coronary atherosclerosis present increased plasma soluble CD163 (sCD163) levels (3).

Monocyte chemotactic protein-1 (MCP-1) has been reported to have a significant role in the pathogenesis of atherosclerosis (4). MCP-1 is produced by different cell types within the arterial wall. In addition to its role in promoting the migration of monocytes into tissues, MCP-1 is involved in the expression of various proinflammatory genes. Increased MCP-1 expression has been immunohistochemically detected in atherosclerotic lesions, implicating this protein in the recruitment of monocyte-macrophages during the atherosclerosis process (5).

Apart from their known hypolipidemic properties, statins also exhibit antiatherosclerotic effects that are cholesterol-lowering independent. The clinical benefits of these agents are correlated with the improvement in endothelial dysfunction, with reduced blood thrombogenicity, as well as with their anti-inflammatory and immunomodulatory activities (6).

Simvastatin is usually co-administered along with the atherogenic diets used in experimental protocols of atherosclerosis in animals or administered following the induction 
of hyperlipidemia (7-9). The aim of the present study was to investigate the potential preventive hypolipidemic activity of simvastatin in rabbits that initially received simvastatin along with normal chow, following which they received an atherogenic diet for the same period.

\section{Materials and methods}

Animals and ethical approval. Twenty-two 2-month-old male New Zealand white rabbits [body weight (mean \pm SD) $3743.3 \pm 631.6$ g; Rabbit Farms, Farma Trompetas, Megara, Greece] were individually housed in stainless steel wire-bottom cages. Animals were kept in a temperature-controlled environment $\left(19 \pm 1^{\circ} \mathrm{C}\right.$; humidity, $\left.55 \pm 5 \%\right)$ under a 12 -h light/dark cycle (5:30 a.m. to 5:30 p.m.) in an air-conditioned room with free access to standard rabbit chow and tap water.

The experimental protocol was reviewed and approved by the Veterinary Directorate of Attica Region and by the Ethics Committee of the Medicine School of the National and Kapodistrian University of Athens (Athens, Greece), in accordance with the EU Legislation for the use of animals for scientific purposes. After 2 weeks of acclimatization, the rabbits were randomly assigned to three groups.

The control group (group C) consisted of six rabbits that received standard commercial rabbit chow. The cholesterol group (group $\mathrm{A} ; \mathrm{n}=8$ ) consisted of rabbits fed with a commercial rabbit chow supplemented with $0.5 \% \mathrm{w} / \mathrm{w}$ cholesterol (a cholesterol diet) for 8 weeks and then with normal chow for a further 8 weeks. In group B $(n=8)$, the rabbits initially received standard commercial rabbit chow along with $3 \mathrm{mg} / \mathrm{kg} /$ day simvastatin (Bennett Pharmaceuticals SA, Kifisia, Greece) in the drinking water for the first 8 weeks and subsequently were fed with a cholesterol-enriched diet for 8 weeks.

Experimental procedure. The rabbit chow [Conigli Svezzamento, S.I.V.A.M. Società Italiana Veterinaria Agricola Milano S.P.A., Casalpusterlengo (LO), Italy] consisted of the following (w/w): $37 \%$ carbohydrates, $16 \%$ proteins, $4 \%$ fat, $15 \%$ fiber, $11 \%$ water, $8 \%$ Ash and an appropriate mixture of minerals and vitamins for the healthy subsistence of the animals in the laboratory (added to the premix by the manufacturer). The high-cholesterol diet was prepared by dissolving the appropriate quantity of cholesterol in diethyl ether (without butylated hydroxytoluene as an inhibitor) and thoroughly coating the pellets of chow with the mixture. After ether evaporation, the special diet was stored at $-20^{\circ} \mathrm{C}$ until use. Fresh food from the freezer was provided to the animals every morning between 8:30 and 10:30 a.m.

The administration of cholesterol through the enrichment of a standard diet was performed according to the widely used method described in rodent models of diet-induced atherosclerosis (10). The statin administration through drinking water is a procedure used in a number of experimental studies with rabbits (11). Furthermore, the quantity of food provided to the rabbits was not restricted, as dietary stress severely affects immune and cell functions in rabbits (12).

Simvastatin treatment. In order to ensure that all simvastatin-treated rabbits received equal doses ( $3 \mathrm{mg} / \mathrm{kg} / \mathrm{day})$, a small volume of water supplemented with $3 \mathrm{mg} / \mathrm{kg}$ simvastatin (based on body weight) was placed in a separate bottle in each rabbit cage on a daily basis, in the morning, while the water bottle without simvastatin was removed during this time. During the following hours, water consumption was regularly checked and when the bottles were empty, the initial bottles, filled with water free of simvastatin, were again placed in the cages.

The body weight of the animals was measured at baseline, and at 8 and 16 weeks. Blood samples (1.5-2 ml) were collected, at baseline, 4,8 and 16 weeks of the experimental period, from the central ear artery without anesthesia after a 14-h fast. At the end of the study, the animals were anesthetized by intramuscular injection of ketamine and xylazine (35 and $10 \mathrm{mg} / \mathrm{kg}$ of body weight, respectively). Subsequent to anesthesia, the rabbits were intravenously administered with an overdose of sodium pentobarbital. The aorta from the arch to the iliac bifurcation and myocardial tissue samples were rapidly removed.

Serum lipids measurements. The blood serum was separated by centrifugation at $1,000-2,000 \mathrm{x}$ g for $10 \mathrm{~min}$ in a refrigerated centrifuge $\left(4^{\circ} \mathrm{C}\right)$ and was stored at $-80^{\circ} \mathrm{C}$ until analysis. Serum concentrations of total cholesterol and of triglycerides were determined at baseline, at the 8th and 16th week of the experimental period, using the enzymatic 4-aminophenazone-phenol commercial kit (Biotechnological Applications, Athens, Greece). High-density lipoprotein (HDL) cholesterol was determined using a cholesterol enzymatic photometric method (HDL cholesterol reagent; Medicon Hellas, Gerakas, Greece) according to the manufacturer's instructions and the low-density lipoprotein (LDL) cholesterol was determined according to the following mathematic model: LDL cholesterol $=$ total cholesterol $-($ HDL cholesterol + triglycerides $/ 5)$. All samples were analyzed at the Laboratory of Experimental Surgery and Research of the Medical School of Athens (Athens, Greece).

MCP-1 and CD163 measurements in serum. MCP-1 and CD163 serum levels were determined in the serum samples collected during the first 4 and 8 weeks of the experimental period, using enzyme-linked immunosorbent assay (ELISA) and commercially available kits, according to the manufacturer's instructions (MCP-1: USCN Life Science Inc., Wuhan, China; CD163: BG Bluegene, Shanghai, China). Samples were run in 96-well plates on a plate reader (Elisa reader BIORAD 680; Bio-Rad Laboratories, Inc., Hercules, CA, USA) to determine absorbance at wavelengths of 450 and $550 \mathrm{~nm}$.

Histological evaluation of aortic and myocardial tissue. The isolated aorta was quickly transferred in ice-cold $0.9 \% \mathrm{w} / \mathrm{v}$ $\mathrm{NaCl}$ solution, cleaned to remove surrounding tissue fragments and sliced open along its length. The vessel was then fixed in neutral $10 \% \mathrm{v} / \mathrm{v}$ formalin. A similar procedure was followed for the myocardial tissue. Two days after fixation in formalin, representative sections of aorta (thoracic and abdominal) and heart were obtained, and processed for paraffin incubation. Sections of $5-\mu \mathrm{m}$ thickness were cut and stained with hematoxylin and eosin for microscopic examination. Image analysis was performed in order to evaluate intimal thickening in aorta 
Table I. Serum lipid and body weight levels. ${ }^{a}$

\begin{tabular}{lcccc}
\hline Parameter & Group & Baseline & 8 weeks & 16 weeks \\
\hline Serum cholesterol & A & $57.00(21.00)^{\mathrm{b}, \mathrm{c}}$ & $1,865.00(580.00)^{\mathrm{c}-\mathrm{f}}$ & $710.00(96.50)^{\mathrm{b}, \mathrm{d}-\mathrm{f}}$ \\
levels (mg/dl) & B & $47.50(10.75)^{\mathrm{b}, \mathrm{c}}$ & $29.00(5.75)^{\mathrm{c}-\mathrm{e}, \mathrm{g}}$ & $111.50(96.75)^{\mathrm{b}, \mathrm{d}, \mathrm{e}}$ \\
& C & $54.00(43.75)$ & $43.00(22.25)^{\mathrm{f}, \mathrm{g}}$ & $52.00(22.25)^{\mathrm{f}}$ \\
Serum high-density lipoprotein & A & $20.00(10.50)^{\mathrm{b}, \mathrm{c}}$ & $23.00(11.00)^{\mathrm{d}}$ & $24.00(10.00)^{\mathrm{d}}$ \\
cholesterol levels (mg/dl) & B & $27.50(8.00)$ & $27.50(8.00)$ & $26.00(3.75)$ \\
& C & $18.00(16.00)$ & $18.50(14.75)$ & $20.00(15.50)$ \\
Serum low-density lipoprotein & A & $24.40(10.80)^{\mathrm{b}, \mathrm{c}, \mathrm{e}}$ & $1,794.20(589.70)^{\mathrm{c}-\mathrm{f}}$ & $660.80(110.80)^{\mathrm{b}, \mathrm{d}-\mathrm{f}}$ \\
cholesterol levels (mg/dl) & B & $8.90(10.60)^{\mathrm{c}, \mathrm{e}, \mathrm{g}}$ & $10.10(10.30)^{\mathrm{c}, \mathrm{e}}$ & $74.60(93.30)^{\mathrm{b}, \mathrm{d}, \mathrm{e}}$ \\
& C & $24.00(29.30)^{\mathrm{g}}$ & $20.10(14.35)^{\mathrm{f}}$ & $27.40(21.65)^{\mathrm{f}}$ \\
Serum triglyceride & A & $55.00(47.50)^{\mathrm{b}, \mathrm{c}}$ & $152.00(77.00)^{\mathrm{d}-\mathrm{f}}$ & $156.00(153.50)^{\mathrm{d}-\mathrm{f}}$ \\
levels (mg/dl) & B & $49.50(17.25)$ & $49.50(18.50)^{\mathrm{e}}$ & $44.50(27.25)^{\mathrm{e}}$ \\
& C & $51.50(22.75)$ & $37.00(21.25)^{\mathrm{f}}$ & $37.50(19.00)^{\mathrm{f}}$ \\
Body weight (g) & A & $3,200.00(495.00)^{\mathrm{c}, \mathrm{e}}$ & $3,850.00(725.00)^{\mathrm{c}, \mathrm{e}}$ & $4,180.00(675.00)^{\mathrm{b}, \mathrm{d}, \mathrm{e}}$ \\
& B & $3,800.00(312.50)^{\mathrm{b}, \mathrm{c}, \mathrm{e}, \mathrm{g}}$ & $4,325.00(377.50)^{\mathrm{c}, \mathrm{d}, \mathrm{e}, \mathrm{g}}$ & $4,425.00(410.00)^{\mathrm{b}, \mathrm{d}, \mathrm{g}}$ \\
& C & $3,250.00(232.50)^{\mathrm{g}}$ & $3,555.00(272.50)^{\mathrm{g}}$ & $4,120.00(362.50)^{\mathrm{g}}$ \\
\hline
\end{tabular}

${ }^{\text {aS }}$ erum lipid levels and body weights among experimental groups at the different time points [values are presented as medians (interquartile range)].Group A, cholesterol group (rabbits received a cholesterol diet for 8 weeks and then normal chow for another 8 weeks); group B (treatment group), animals received standard rabbit chow along with simvastatin ( $3 \mathrm{mg} / \mathrm{kg} / \mathrm{day})$ for 8 weeks followed by a high cholesterol diet for another 8 weeks; group $\mathrm{C}$, control group. ${ }^{\mathrm{b}} \mathrm{P}<0.05$ vs. 8 weeks; ${ }^{\mathrm{C}} \mathrm{P}<0.05$ vs. 16 weeks; ${ }^{\mathrm{d}} \mathrm{P}<0.05$ vs. baseline; ${ }^{\mathrm{e}} \mathrm{P}<0.05$, A vs. $\mathrm{B}$; ${ }^{\mathrm{f}} \mathrm{P}<0.05$ A vs. C; ${ }^{g} \mathrm{P}<0.05$ B vs. C.

specimens of aortic, and myocardial tissues. Slides were digitized using a microscope eclipse 80i (Nikon Corporation, Tokyo, Japan) attached to a digital camera (DS-2 MW; Nikon Corporation). The images were transferred to a computer equipped with Image ProPlus version 5.1 (Media Cybernetics, Inc., Rockville, MD, USA) and the layers of the vessel wall (intima, media and serosa) were traced. The intimal thickening was then calculated semi-automatically.

The evaluation of myocardial damage and atherosclerotic alterations was qualitative and was performed by two independent pathologists. The atherosclerotic damage in the aortic tissue was classified into three groups according to the grade of alteration severity as follows: Grade 1, mild; grade 2, moderate; and grade 3 , severe $(13,14)$. The classification of the three groups depended on the thickness of the atherosclerotic plaque/lumen stenosis and the perimetrical area of the vessel occupied by the plaque. The histopathological results were consistent with the observations of the two independent pathologists. The pathologists were absolutely blind to the experimental treatment.

Statistical analysis. Data are expressed as medians (interquartile range). Comparisons between more than two groups were performed using Kruskal-Wallis's test while the Mann-Whitney U test was used for post hoc/multiple comparisons. Comparisons between more than two measurements of the same group were performed using Friedman's test and Wilcoxon's signed rank test as a post hoc test. The Fisher's exact test was used in order to analyze the data from the histopathology. All tests were two-sided. $\mathrm{P}<0.05$ was considered to indicate a statistically significant difference and analyses were conducted using SPSS statistical software version 19.0 (SPSS, Inc., Chicago, IL, USA).

\section{Results}

Serum lipid levels. At baseline, total serum cholesterol and LDL cholesterol levels were similar between the three study groups $(\mathrm{P}>0.05)$. Total cholesterol and LDL cholesterol levels were significantly increased in group A from baseline to 8 weeks ( $\mathrm{P}=0.043$ for total and LDL cholesterol levels), and subsequently a significant reduction was identified from 8 to 16 weeks ( $\mathrm{P}=0.043$ for total and LDL cholesterol levels). No significant changes were found in total cholesterol and LDL cholesterol levels during the follow up for group C. In group $\mathrm{B}$, the total cholesterol levels decreased from baseline to 8 weeks $(\mathrm{P}=0.012)$ and subsequently a significant increase was observed for the total cholesterol $(\mathrm{P}=0.012)$ and $\mathrm{LDL}$ cholesterol levels $(\mathrm{P}=0.017)$ from 8 to 16 weeks. Regarding serum total and LDL cholesterol levels, the majority of group comparisons were significant at 8 weeks (total cholesterol levels: A vs. $\mathrm{B}, \mathrm{P}=0.002$; $\mathrm{A}$ vs. $\mathrm{C}, \mathrm{P}=0.011$; $\mathrm{B}$ vs. $\mathrm{C}, \mathrm{P}=0.017$; LDL cholesterol levels: A vs. $\mathrm{B}, \mathrm{P}=0.002$; A vs. $\mathrm{C}, \mathrm{P}=0.011$ ), not including groups $\mathrm{C}$ and $\mathrm{B}$ that had similar LDL cholesterol levels. At 16 weeks, groups $\mathrm{C}$ and B had similar total cholesterol and LDL cholesterol levels, while group A had higher total cholesterol and LDL cholesterol levels when compared with groups $\mathrm{C}(\mathrm{P}=0.014$ for total cholesterol and LDL cholesterol levels) and $\mathrm{B}(\mathrm{P}=0.003$ for total cholesterol levels and LDL cholesterol levels; Table I and Fig. 1).

Triglyceride levels were significantly increased in group A from baseline to 8 weeks $(\mathrm{P}=0.043)$. At 8 and 16 weeks, group A had higher triglyceride levels when compared with groups $\mathrm{C}$ and $\mathrm{B}$ ( 8 weeks: A vs. $\mathrm{B}, \mathrm{P}=0.039$; A vs. $\mathrm{C}, \mathrm{P}=0.019$; 16 weeks: A vs. $B, P=0.003$; A vs. $C, P=0.014$; Table I and Fig. 1). 

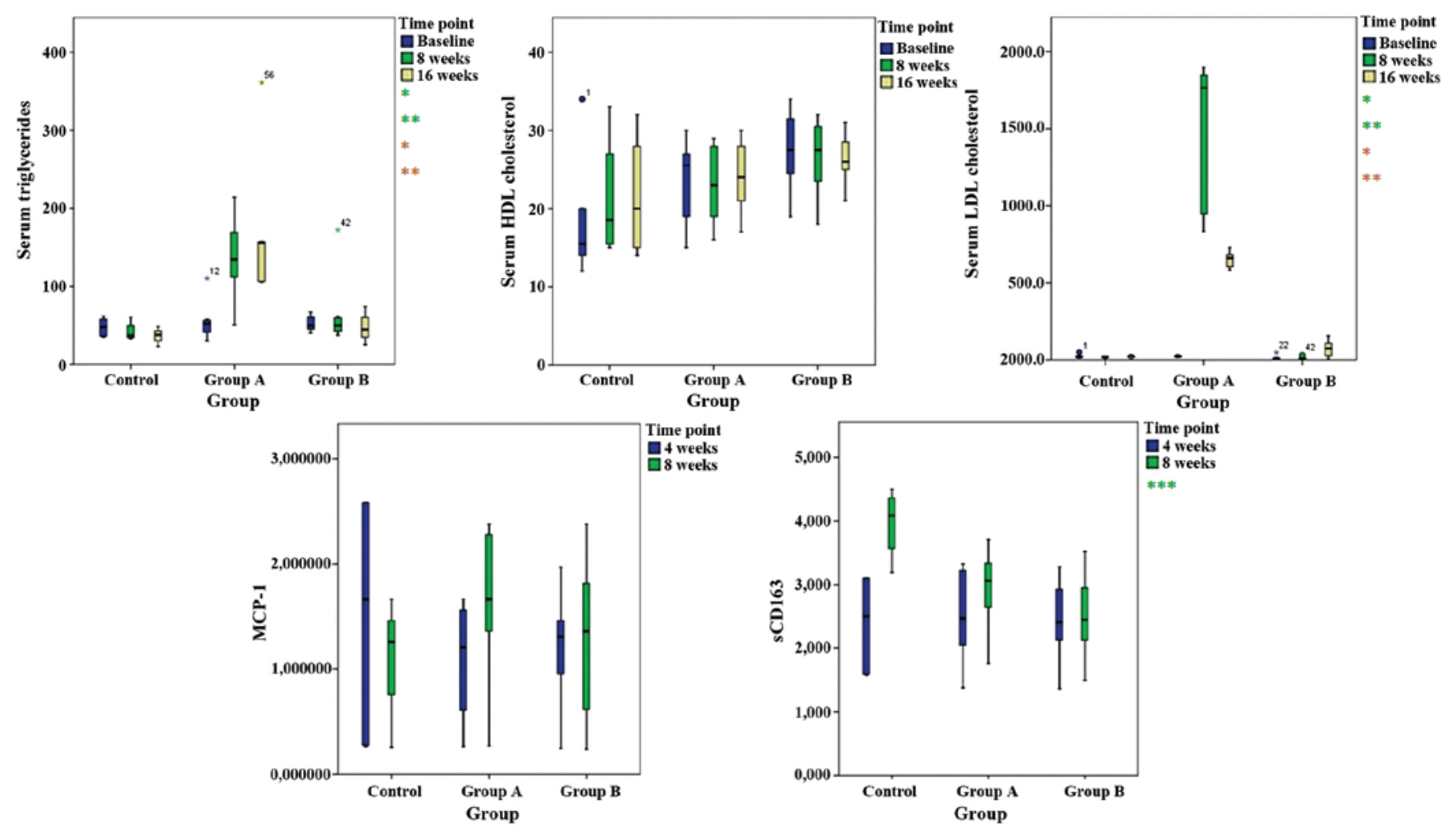

Figure 1. Serum lipid levels, serum MCP-1 and sCD163 levels. Box plots displaying the second and third quartiles, as well as the median, of serum triglyceride (mg/dl), HDL and LDL cholesterol (mg/dl), MCP-1 (ng/ml) and sCD163 (ng/ml) levels among experimental groups at the different time points. Upon completion of the study (16 weeks) the box plots indicate that rabbits in group B (simvastatin pre-treatment, $3 \mathrm{mg} / \mathrm{kg} / \mathrm{day}$ ) had reduced LDL cholesterol levels when compared with the atherogenic-fed group A, while group B did not differ to the control group. MCP-1, monocyte chemotactic protein-1; HDL, high-density lipoprotein; LDL, low-density lipoprotein. ${ }^{*} \mathrm{P}<0.05$, A vs. $\mathrm{B} ;{ }^{* *} \mathrm{P}<0.05 \mathrm{~A}$ vs. control; ${ }^{* * *} \mathrm{P}<0.05 \mathrm{~B}$ vs. control.

At 8 weeks, group A rabbits had lower body weights when compared with rabbits in group $\mathrm{B}(\mathrm{P}=0.002)$, while group $\mathrm{C}$ rabbits had lower body weights when compared with those in group $\mathrm{B}(\mathrm{P}=0.006)$. Body weight was increased from baseline to 8 weeks for group $\mathrm{B}(\mathrm{P}=0.012)$ and from baseline to 16 weeks for groups $\mathrm{A}(\mathrm{P}=0.043)$ and $\mathrm{B}(\mathrm{P}=0.012)$. All values and significant differences between groups and between different time points are presented in Table I.

Serum MCP-1 and sCD163 levels. The MCP-1 mean values are presented in Table II. MCP-1 values did not differ significantly among groups regarding the two measurements. Although no significant changes were detected in rabbit MCP-1 values between the 4th and 8th week, a trend towards increase was observed over time, in the mean MCP-1 levels in group A (1.08 vs. $1.56 \mathrm{ng} / \mathrm{ml}$ for 4 th and 8th week MCP-1 levels, respectively; Table II and Fig. 1).

Between 4 and 8 weeks, there were no significant changes in rabbit sCD163 values at the two time points (Table III). At 4 weeks there were no significant differences in sCD163 values among all study groups. At 8 weeks, group $\mathrm{C}$ had significantly higher sCD163 values than group $\mathrm{B}(\mathrm{P}=0.011$; Table III and Fig. 1).

Histopathological analysis. In groups $\mathrm{C}$ and $\mathrm{B}$ all rabbits exhibited normal aortic tissue architecture while $40 \%$ of the rabbits in group A presented atherosclerotic alterations in the aorta (grade 1-3). However, the observed differences among groups (groups C and B vs. group A) did not reach statistical significance (Table IV and Fig. 2). No alterations were observed in the myocardial tissue samples of all groups.

\section{Discussion}

Hypercholesterolemia in combination with increased LDL cholesterol levels represent dominant risk factors for the development and progression of atherosclerosis and consequently of cardiovascular disease (CVD) $(15,16)$. Dyslipidemia, characterized by raised levels of atherogenic lipoproteins are recognized as major risk factors for the development of atherosclerotic CVD (17).

Rabbit is the most common animal model for evaluating the pathophysiology of atherosclerosis. In addition, it is extensively used in experimental protocols investigating the beneficial action of hypolipidemic and anti-inflammatory agents in atherosclerotic processes. The induction of atherosclerosis in rabbits is easily achieved after feeding the animals a high-cholesterol diet, which is not the case in other animal species, such as the wild type C57bl/6 mice that develop hypercholesterolemia with no atherosclerotic plaque formation (18). Various knockout rodent models have been described in the field of spontaneous atherosclerosis, without the need for dietary intervention (including apolipoprotein $\mathrm{E}^{-/-}$and LDL-r $^{-/}$) (19). However, rodent models suffer from disadvantages, which are associated with various discrepancies from the pathophysiologic processes of the disease in humans. Rabbits are particularly sensitive to cholesterol-enriched diets accumulating large quantities of cholesterol in their plasma, thus, 
Table II. Serum MCP-1 levels [median (interquartile range)] at the 4th and 8th week of the study.

\begin{tabular}{lrr}
\hline & \multicolumn{2}{c}{ Serum MCP-1 levels (ng/ml) } \\
\cline { 2 - 3 } Group & 4 weeks & 8 weeks \\
\hline A & $1.20(1.12)$ & $1.66(1.21)$ \\
B & $1.30(0.56)$ & $1.36(1.39)$ \\
C & $1.66(2.31)$ & $1.25(1.05)$ \\
\hline & & \\
Group A, cholesterol group (rabbits & received a cholesterol diet \\
for 8 weeks and then normal chow for another 8 weeks); group B \\
(treatment group), animals received standard rabbit chow along with \\
simvastatin (3 mg/kg/day) for 8 weeks followed by a high cholesterol \\
diet for another 8 weeks; group C, control group. No significant dif- \\
ferences were identified between groups A and B, A and C or B and C. \\
$\begin{array}{l}\text { Furthermore, no significant differences were identified between } \\
\text { weeks } 4 \text { and } 8 \text { in any of the groups. MCP-1, monocyte chemotactic }\end{array}$ \\
protein-1.
\end{tabular}

Table III. Serum sCD163 levels [median (interquartile range)] at the 4th and 8th week of the study.

\begin{tabular}{llc}
\hline & \multicolumn{2}{c}{ Serum sCD163 levels $(\mathrm{ng} / \mathrm{ml})$} \\
\cline { 2 - 3 } Group & 4 weeks & 8 weeks \\
\hline A & $2.46(1.24)^{\mathrm{a}}$ & $3.06(1.01)$ \\
B & $2.40(0.88)$ & $2.45(0.91)^{\mathrm{b}}$ \\
C & $2.50(1.51)$ & $4.08(1.05)^{\mathrm{c}}$ \\
\hline
\end{tabular}

Group A, cholesterol group (rabbits received a cholesterol diet for 8 weeks and then normal chow for another 8 weeks); group B (treatment group), animals received standard rabbit chow along with simvastatin ( $3 \mathrm{mg} / \mathrm{kg} /$ day) for 8 weeks followed by a high cholesterol diet for another 8 weeks; group $\mathrm{C}$, control group. ${ }^{\mathrm{a}} \mathrm{P}=0.043$ vs. 8 weeks; ${ }^{b} \mathrm{P}=0.011$ vs. $\mathrm{C} ;{ }^{\mathrm{c}} \mathrm{P}=0.011$ vs. $\mathrm{B}$. sCD163, soluble $\mathrm{CD} 163$.

simulating the course of human atherosclerotic disease (20). Furthermore, the extent of the lesions developed in the aortic wall in rabbits is proportional to the quantity of cholesterol consumed (21).

Statins have been extensively investigated for their hypolipidemic and antiatherosclerotic potential (22). Based on the findings of numerous clinical studies $(23,24)$, statins have been proposed to be particularly effective, and constitute one of the most critical treatment options against atherogenic dyslipidemia (25). Simvastatin is a 3-hydroxy-3-methylglutaryl-coenzyme A reductase inhibitor, known to reduce serum lipid levels $(26,27)$. A number of studies have been conducted to elucidate the underlying mechanisms involved in the beneficial action of statins against the induction of hyperlipidemia and the process of atherogenesis $(13,28-30)$. Statins improve endothelial function, diminish oxidative stress and platelet adhesion and enhance atherosclerotic plaque stability (31). The implicated mechanistic pathways, depending on the type of statin used, continue to be investigated.
Table IV. Histopathological analysis of aorta and heart tissue samples. ${ }^{2}$

\begin{tabular}{lcrrr}
\hline & & \multicolumn{3}{c}{ Group } \\
\cline { 3 - 5 } Atherosclerotic damage & Grade & $\mathrm{A}(\%)$ & $\mathrm{B}(\%)$ & $\mathrm{C}(\%)$ \\
\hline Aorta & 0 & 60.00 & 100.00 & 100.00 \\
& $1-3$ & 40.00 & 0.00 & 0.00 \\
Heart & 0 & 100.00 & 100.00 & 100.00 \\
& $1-3$ & 0.00 & 0.00 & 0.00 \\
\hline
\end{tabular}

${ }^{a}$ The atherosclerotic damage is presented as scores (grade 1-3). Group A, cholesterol group (rabbits received a cholesterol diet for 8 weeks and then normal chow for another 8 weeks); group B (treatment group), animals received standard rabbit chow along with simvastatin ( $3 \mathrm{mg} / \mathrm{kg} /$ day) for 8 weeks followed by a high cholesterol diet for another 8 weeks; group C, control group. No significant differences were recorded in group A when comparing the percentage of animals that showed signs of atherosclerotic damage in the aorta during histopathologival analysis, with the percentage of animals that showed damage in the heart (Fisher's exact test; $\mathrm{P}=0.444$ ). No significant differences were observed between groups $\mathrm{A}-\mathrm{C}$ in the percentage of animals that showed atherosclerotic damage in the aorta $(\mathrm{P}>0.05)$.

In a previous clinical study, CVD patients were treated either with simvastatin or atorvastatin for a 4-week period. Blood lipid levels were improved in the two groups of patients, while mean platelet volume levels (an indicator of platelet activation) significantly decreased in all patients (32). In another study investigating the effects of simvastatin or pravastatin administration in hypercholesterolaemic rabbits, it was observed that only pravastatin decreased the size of the infarction; an effect that is possibly associated with reduced nitrotyrosine and the increased endothelial NO synthase levels measured in the myocardial tissue (33). The same conclusions regarding the role of simvastatin on the size of infarcts in hyperlipidemic rabbits, through the attenuation of oxidative stress, have been reported in a study by Iliodromitis et al (34).

Animals in group A exhibited increased serum total cholesterol and LDL cholesterol levels at week 8 and at the end of the experimental protocol as compared with the baseline measurements for this group.

Notably, at the end of the experimental period, in the group B rabbits, which received simvastatin during the first 8 weeks of the study, the serum total and LDL cholesterol levels were lower than the levels recorded in the rabbits from goup A, although they did not differ when compared with the respective lipid levels of rabbits in the control group. This observation supports the hypothesis that simvastatin exerts a preventive role against diet-induced hypercholesterolemia.

At the end of the study, the animals that were treated with simvastatin before administration of a cholesterol-enriched diet, presented reduced triglyceride levels as compared with the untreated group. In addition to their total cholesterol- and LDL cholesterol-lowering effects, statins are effective in improving hypertriglyceridemia (35). Although fibrates remain the most important treatment for patients suffering from triglyceride levels $>500 \mathrm{mg} / \mathrm{dl}$, statins are considered as first-line treatment 

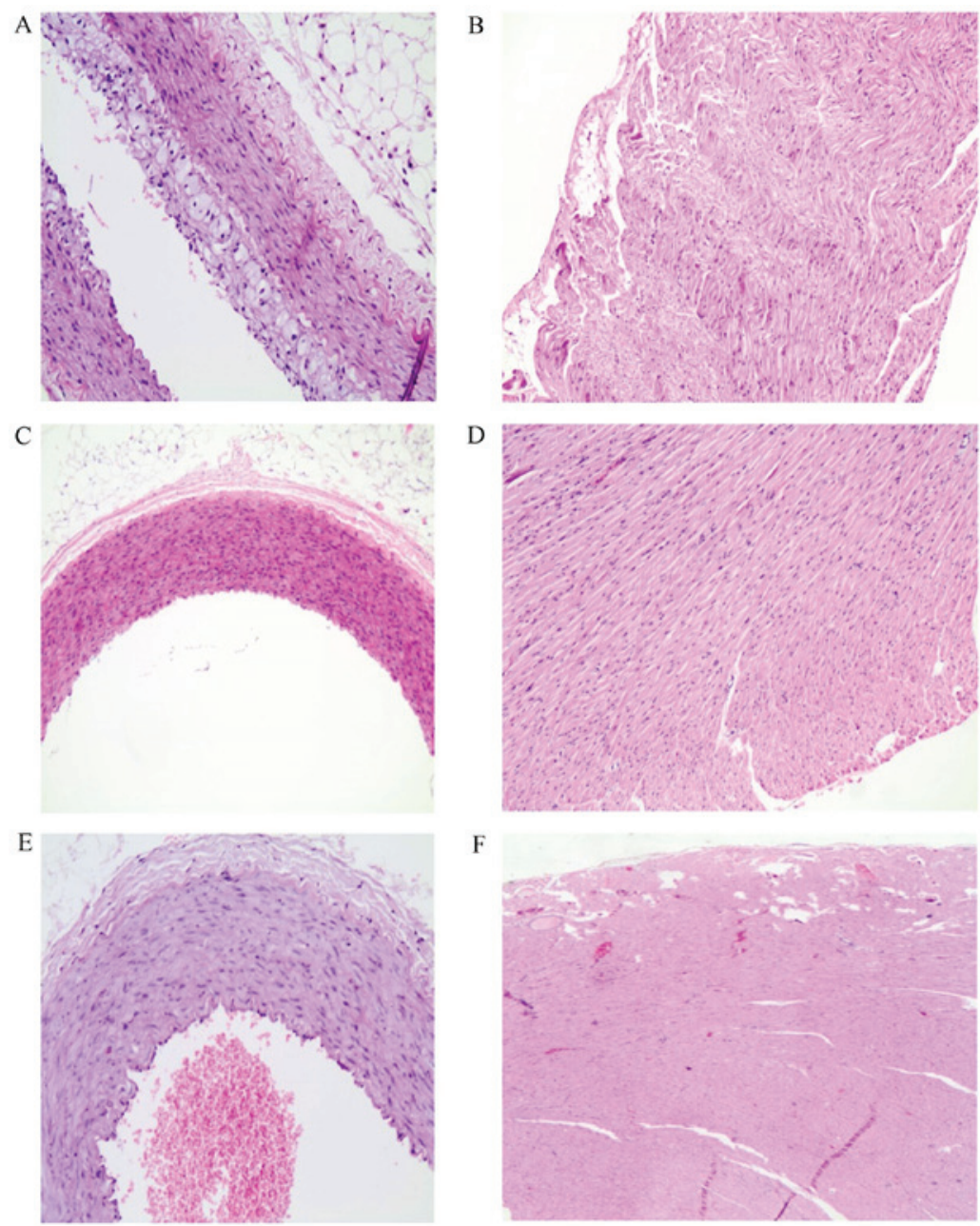

Figure 2. Histopathological analysis in aortas and myocardial tissue samples. Hematoxylin and eosin-stained sections of (A) atheromatous aorta and (B) normal myocardium obtained from rabbits in group A (magnification, x100). Hematoxylin and eosin-stained sections of normal (C) aorta and (D) myocardium from group B rabbits (magnification, x100). No atherosclerotic lesions were observed in the aortic wall or the myocardium of any of the rabbits subsequent to prophylactic administration of simvastatin (3 mg/kg/day). Normal (E) aorta and (F) myocardium samples obtained from group C (magnification, x100)

for those with high triglyceride levels, which are maintained below this threshold (36).

The histological examination of the aortic tissue samples was consistent with the results obtained from the evaluation of the serum lipid profile of the rabbits. More precisely, simvastatin pre-treatment prevented the cholesterol diet-induced atherosclerotic damage in aortas.

The increased permeability of the endothelium, developed in the early atherosclerotic process, enhances the migration of different types of leukocytes to the aortic wall $(37,38)$. Although various chemotactic substances induce monocyte migration, MCP-1 constitutes the major regulator for the recruitment of monocytes into the subendothelial cell layer (39). The monocytes adhere to the epithelium, move to the intima, then transform to foam cells and gradually to fatty streak, which is the first grossly visible lesion in the development of atherosclerosis (37).

In a study where rabbits were fed with a cholesterol-enriched diet for a six-week period, the total cholesterol levels were significantly increased from the third day of the experimental procedure (40). However, molecular analysis of the aortic tissue of the animals revealed that MCP-1 mRNA levels remained unchanged in the early stages of the atherogenetic process, while raised MCP-1 mRNA expression levels were detected only after three weeks under high cholesterol feeding. This implies that the long-term consequences, due to the increased serum lipid levels, stimulate the alterations in MCP-1 expression (40).

In the present study, differences in MCP-1 levels among the experimental groups were not detected at 4 and 8 weeks, or between the measurements of each animal group at the different time points, although certain trends were observed. The levels of MCP-1 in the untreated rabbits (group A) exhibited an increasing trend at 8 weeks, although this observation was not statistically significant. Therefore, MCP-1 may be essential in the process of monocyte migration in the arterial wall during the process of atherogenesis in these animals.

sCD163 constitutes a marker of monocyte/macrophage activation. In a study investigating HIV-infected patients, the sCD163 concentration was associated with certain characteristics of atherosclerotic plaque, such as the total number of non-calcified segments and the percentage of non-calcified plaques (41). In another study, increased sCD163 levels were detected in treated HIV-infected men and these were associated with the extent of atherosclerotic damage (42). 
A significant increase in sCD163 levels of rabbits in group A was recorded at 8 weeks when compared with the respective levels at 4 weeks. Notably, a decrease in sCD163 serum levels was not detected in the group B rabbits that were preventively treated with simvastatin.

The findings of the current study are, however, partly limited, as the hepatic enzyme activities in the rabbits were not measured; therefore, potential side effects resulting from the administration of simvastatin were not observed (43).

The present findings indicate that simvastatin pre-treatment, before enrichment of the diet with cholesterol in rabbits, has beneficial hypolipidemic and antiatherosclerotic effects by inhibiting the increase of total cholesterol, LDL cholesterol and triglyceride levels in the serum. Therefore preventing the formation of atherosclerotic lesions in the thoracic aorta of the rabbits

Regarding implementations for current clinical practice and future research, simvastatin pretreatment appears to exert preventive hypolipidemic activity in rabbits that are due to receive a high cholesterol atherogenic diet. This observation is particularly important, as it provides evidence for the beneficial mode of action of this therapeutic agent. The prevention of atherosclerosis is considered to be crucial in modern societies that, generally, are experiencing epidemical increases in obesity and metabolic syndrome $(44,45)$. Future clinical studies are required to support the current findings and to provide guidelines for future clinical practice.

In conclusion, the findings of the present study indicate for the first time, to the best of our knowledge, that simvastatin may present as an effective strategy for CVD prevention. However, the molecular pathways involved in the prophylactic action of simvastatin against the hypercholesterolemia and the atherogenesis require further investigation. Furthermore, improved understanding and increased scientific evidence of the preventive contribution of simvastatin on the inhibition of the progression of atherosclerosis are required.

\section{Acknowledgements}

The authors would like to thank Mrs. Esmeralda Ntousi, Mr. Panagiotis Tsakiropoulos and Mr. Nikolaos Tsakiropoulos for their assistance during the experimental procedures.

\section{References}

1. Rafieian-Kopaei M, Setorki M, Doudi M, Baradaran A and Nasri H: Atherosclerosis: Process, indicators, risk factors and new hopes. Int J Prev Med 5: 927-946, 2014.

2. Fabriek BO, Dijkstra CD and van den Berg TK: The macrophage scavenger receptor CD163. Immunobiology 210: 153-160, 2005.

3. Aristoteli LP, Møller HJ, Bailey B, Moestrup SK and Kritharides L: The monocytic lineage specific soluble CD163 is a plasma marker of coronary atherosclerosis. Atherosclerosis 184: 342-347, 2006.

4. Lin J, Kakkar V and Lu X: Impact of MCP-1 in atherosclerosis. Curr Pharm Des 20: 4580-4588, 2014.

5. Ylä-Herttuala S, Lipton BA, Rosenfeld ME, Särkioja T, Yoshimura T, Leonard EJ, Witztum JL and Steinberg D: Expression of monocyte chemoattractant protein 1 in macrophage-rich areas of human and rabbit atherosclerotic lesions. Proc Natl Acad Sci USA 88: 5252-5256, 1991.

6. Blanco-Colio LM, Tuñón J, Martín-Ventura JL and Egido J: Anti-inflammatory and immunomodulatory effects of statins. Kidney Int 63: 12-23, 2003.

7. Risovic V, Man D, Sivak O, Lee SD and Wasan KM: Assessing lipid lowering and plasma cholesteryl ester transfer protein activity of simvastatin following administration to rabbits fed a high fat/cholesterol diet. Drug Dev Ind Pharm 32: 609-615, 2006.
8. Zhang YH, Zhang Y, Li J, Tong WX and Xu FQ: Protective effects of Xiongshao Capsule () on anti-inflammatory function of high-density lipoprotein in atherosclerosis rabbit model. Chin J Integr Med 10: 10, 2015.

9. Djerrou Z: Anti-hypercholesterolemic effect of Pistacia lentiscus fatty oil in egg yolk-fed rabbits: A comparative study with simvastatin. Chin J Nat Med 12: 561-566, 2014.

10. Rasmusen C, Moinard C, Martin C, Tricottet V, Cynober L and Couderc R: L-arginine plus atorvastatin for prevention of atheroma formation in genetically hypercholesterolaemic rabbits. Br J Nutr 97: 1083-1089, 2007.

11. Yanni AE, Yatzidis HA, Kavantzas NG, Agapitos EV, Perrea DN and Karayannacos PE: Dietary L-aspartate and L-glutamate inhibit fatty streak initiation in cholesterol-fed rabbit. Nutr Metab Cardiovasc Dis 13: 80-86, 2003.

12. Franci O, Amici A, Margarit R, Merendino N and Piccolella E: Influence of thermal and dietary stress on immune response of rabbits. J Anim Sci 74: 1523-1529, 1996.

13. Cook DL, Mills LM and Green DM: The mechanism of alloxan protection in experimental atherosclerosis. J Exp Med 99: $119-124,1954$

14. Kamimura R, Suzuki S, Sakamoto H, Miura N, Misumi K and Miyahara K: Development of atherosclerotic lesions in cholesterol-loaded rabbits. Exp Anim 48: 1-7, 1999.

15. Verma DR and Brinton EA: Management of hypercholesterolemia for prevention of atherosclerotic cardiovascular disease: Focus on the potential role of recombinant anti-PCSK9 monoclonal antibodies. Rev Cardiovasc Med 15: 86-101, quiz 101, 2014.

16. Roberts WC: Twenty questions on atherosclerosis. Proc (Bayl Univ Med Cent) 13: 139-143, 2000.

17. Roh E, Ko SH, Kwon HS, Kim NH, Kim JH, Kim CS, Song KH, Won JC, Kim DJ, Choi SH, et al; Taskforce Team of Diabetes Fact Sheet of the Korean Diabetes Association: Prevalence and Management of Dyslipidemia in Korea: Korea National Health and Nutrition Examination Survey during 1998 to 2010. Diabetes Metab J 37: 433-449, 2013.

18. Korou LM, Agrogiannis G, Koros C, Kitraki E, Vlachos IS, Tzanetakou I, Karatzas T, Pergialiotis V, Dimitroulis D and Perrea DN: Impact of N-acetylcysteine and sesame oil on lipid metabolism and hypothalamic-pituitary-adrenal axis homeostasis in middle-aged hypercholesterolemic mice. Sci Rep 4: 6806, 2014

19. Kapourchali FR, Surendiran G, Chen L, Uitz E, Bahadori B and Moghadasian MH: Animal models of atherosclerosis. World J Clin Cases 2: 126-132, 2014.

20. Dornas WC, Oliveira TT, Augusto LE and Nagem TJ: Experimental atherosclerosis in rabbits. Arq Bras Cardiol 95: 272-278, 2010 (In Portuguese).

21. Yanni AE: The laboratory rabbit: An animal model of atherosclerosis research. Lab Anim 38: 246-256, 2004.

22. Profumo E, Buttari B, Saso L and Rigano R: Pleiotropic effects of statins in atherosclerotic disease: Focus on the antioxidant activity of atorvastatin. Curr Top Med Chem 14: 2542-2551, 2014.

23. Tousoulis D, Oikonomou E, Economou EK, Crea F and Kaski JC: Inflammatory cytokines in atherosclerosis: Current therapeutic approaches. Eur Heart J 37: 1723-1732, 2016.

24. März W, Scharnagl H, Gouni-Berthold I, Silbernagel G, Dressel A, Grammer TB, Landmesser U, Dieplinger H, Windler E and Laufs U: LDL-Cholesterol: Standards of Treatment 2016: A German Perspective. Am J Cardiovasc Drugs 16: 323-336, 2016.

25. Ahn $\mathrm{CH}$ and Choi $\mathrm{SH}$ : New drugs for treating dyslipidemia: Beyond statins. Diabetes Metab J 39: 87-94, 2015.

26. Toth PP: Drug treatment of hyperlipidaemia: A guide to the rational use of lipid-lowering drugs. Drugs 70: 1363-1379, 2010.

27. Toth PP and Davidson MH: Simvastatin plus ezetimibe: Combination therapy for the management of dyslipidaemia. Expert Opin Pharmacother 6: 131-139, 2005.

28. Satoh M, Takahashi Y, Tabuchi T, Minami Y, Tamada M, Takahashi K, Itoh T, Morino Y and Nakamura M: Cellular and molecular mechanisms of statins: An update on pleiotropic effects. Clin Sci (Lond) 129: 93-105, 2015.

29. Tissier F, Mallem Y, Goanvec C, Didier R, Aubry T, Bourgeois N, Desfontis JC, Dubreuil M, Le Grand Y, Mansourati J, et al: A non-hypocholesterolemic atorvastatin treatment improves vessel elasticity by acting on elastin composition in WHHL rabbits. Atherosclerosis 251: 70-77, 2016

30. Lin CP, Huang PH, Lai CF, Chen JW, Lin SJ and Chen JS: Simvastatin Attenuates Oxidative Stress, NF- $\kappa$ B Activation, and Artery Calcification in LDLR-/- Mice Fed with High Fat Diet via Down-regulation of Tumor Necrosis Factor- $\alpha$ and TNF Receptor 1. PLoS One 10: e0143686, 2015. 
31. Ludman A, Venugopal V, Yellon DM and Hausenloy DJ: Statins and cardioprotection - more than just lipid lowering? Pharmacol Ther 122: 30-43, 2009.

32. Xian-Yu JB, Feng JF, Chen YC and Yang YW: Effects of simvastatin and atorvastatin on biochemical and hematological markers in patients with risk of cardiovascular diseases. Int $\mathbf{J}$ Clin Exp Med 8: 13983-13989, 2015.

33. Andreadou I, Farmakis D, Prokovas E, Sigala F, Zoga A Spyridaki K, Papalois A, Papapetropoulos A, Anastasiou-Nana M, Kremastinos DT, et al: Short-term statin administration in hypercholesterolaemic rabbits resistant to postconditioning: Effects on infarct size, endothelial nitric oxide synthase, and nitro-oxidative stress. Cardiovasc Res 94: 501-509, 2012.

34. Iliodromitis EK, Andreadou I, Prokovas E, Zoga A, Farmakis D, Fotopoulou T, Ioannidis K, Paraskevaidis IA and Kremastinos DT: Simvastatin in contrast to postconditioning reduces infarct size in hyperlipidemic rabbits: Possible role of oxidative/nitrosative stress attenuation. Basic Res Cardiol 105: 193-203, 2010.

35. Stein EA, Lane M and Laskarzewski P: Comparison of statins in hypertriglyceridemia. Am J Cardiol 81 (4A): 66B-69B, 1998.

36. Brinton EA: Management of hypertriglyceridemia for prevention of atherosclerotic cardiovascular disease. Cardiol Clin 33: 309-323, 2015

37. Ross R: Atherosclerosis - an inflammatory disease. N Engl J Med 340: 115-126, 1999.

38. Deshmane SL, Kremlev S, Amini S and Sawaya BE: Monocyte chemoattractant protein-1 (MCP-1): An overview. J Interferon Cytokine Res 29: 313-326, 2009.
39. Takahashi K, Takeya M and Sakashita N: Multifunctional roles of macrophages in the development and progression of atherosclerosis in humans and experimental animals. Med Electron Microsc 35: 179-203, 2002.

40. Chen YL, Chang YJ and Jiang MJ: Monocyte chemotactic protein-1 gene and protein expression in atherogenesis of hypercholesterolemic rabbits. Atherosclerosis 143: 115-123, 1999.

41. Burdo TH, Lo J, Abbara S, Wei J, DeLelys ME, Preffer F Rosenberg ES, Williams KC and Grinspoon S: Soluble CD163, a novel marker of activated macrophages, is elevated and associated with noncalcified coronary plaque in HIV-infected patients. J Infect Dis 204: 1227-1236, 2011.

42. McKibben RA, Margolick JB, Grinspoon S, Li X, Palella FJ Jr, Kingsley LA, Witt MD, George RT, Jacobson LP, Budoff M, et al: Elevated levels of monocyte activation markers are associated with subclinical atherosclerosis in men with and those without HIV infection. J Infect Dis 211: 1219-1228, 2015.

43. Calderon RM, Cubeddu LX, Goldberg RB and Schiff ER: Statins in the treatment of dyslipidemia in the presence of elevated liver aminotransferase levels: A therapeutic dilemma. Mayo Clin Proc 85: 349-356, 2010.

44. Landecho MF, Moncada R, Valentí V and Frühbeck G: Cardiovascular Prevention in Obese Patients. Curr Pharm Des 22: 22, 2016.

45. Lim S and Eckel RH: Pharmacological treatment and therapeutic perspectives of metabolic syndrome. Rev Endocr Metab Disord 15: 329-341, 2014. 